\title{
Brief review on most advanced detection methods used for Clostridium botulinum neurotoxins (BoNTs) analysis
}

\begin{abstract}
The neurotoxins produced by Clostridium botulinum are the most potent acute toxins known and are the causative agents of the neuroparalytic disease botulism. The toxins act primarily at peripheral cholinergic synapses by blocking the evoked release of the neurotransmitter acetylcholine. This bacteria produces seven types of toxins (A - G) known as Clostridium botulinum neurotoxins (BoNTs). Clostridium botulinum neurotoxins (BoNTs) affects humans, all warm-blooded animals, and fishes due to consumption of contaminated silage, water and canned foods like meat, milk, fruits and vegetables. There are seven distinct serotypes of toxin and their most active forms exist as dichain molecules in which a heavy $(\mathrm{H})$ chain is linked by disulphide bonding to a light $(\mathrm{L})$ chain. The $\mathrm{H}$ chain is believed to be associated with the highly specific and avid binding of toxin to the motor nerve end plates. The toxic activity mostly appears to be associated with the L chain which blockades the calcium-mediated release of acetylcholine by interfering at the molecular level with the mechanisms whereby neurotransmitter-containing vesicles merge with the plasmalemma. There are not any reliable and effective clinical management to avoid the lethal effects of BoNTs, so, its most potent detection can be helpful for developing its cost effective management and timely prevention in susceptible and infected population. Various investigated diagnostic methods are based on various clinical signs and laboratory examinations like, ELISA and culture for isolation of bacteria which used for its analysis in biological samples so that we can prevent havoc of its outbreak as well as timely treatment and prevention.
\end{abstract}

Keywords: Clostridium botulinum, Clostridium botulinum neurotoxins, BoNTs, botulism
Volume 8 Issue 3 - 2019

\author{
Kirti Rani \\ Amity Institute of Biotechnology, Amity University India
}

Correspondence: Kirti Rani,Assistant Professor (III), Amity Institute of Biotechnology, Amity University Uttar Pradesh, Noida, Sec-125, Gautam Buddha Nagar, Noida-201313 (UP), India, Tel +91-1 20-4392946, +91-9990329492,

Email krsharma@amity.edu, Kirtisharma2k@rediffmail.com

Received: March 25, 2019 | Published: May 02, 2019

\section{Introduction}

Botulism is neuroparalytic disease which affects human and animal population. It is caused neurotoxins called botulinum neurotoxins (BoNTs) which produced by gram positive, anaerobic, spore-forming microbes named Clostridium botulinum. It causes flaccid paralysis in infected animals and human population. Because of its bio-warfare agent and potent toxin BoNTs causes disorder in living beings. ${ }^{1}$ BoNTs infection commonly occurs via the ingestion of contaminated food and water due to poor hygiene and may occur from infected weapons in military areas which can cause muscle paralysis or even death in exposed animals and humans. ${ }^{2,3}$ There are no as such geographical boundaries for botulism because of its infrequent occurrence of outbreaks. Reported main route of transmission is by oral ingestion and exposure of wounds with spores of Clostridium botulinum. Clinical symptoms of botulism occur within 24 hour of exposure that lasts up to 17 days in the exposed and susceptible population. Botulinum neurotoxins (BoNTs) contains zinc endopeptidase which blocks binding of vesicles of acetylcholine with the terminal membrane of the motor neuron that lead to flaccid muscle paralysis with lateral recumbency due to muscle weakness and dysphagia. And, in severe cases, it becomes lethal and results in death due to respiratory arrest and pharyngeal paralysis. ${ }^{4,5}$ Botulinum neurotoxin has simple dichain polypeptide that consists of a $100 \mathrm{kDa}$ heavy chain joined by a single disulfide bond to a $50 \mathrm{kDa}$ light chain. The toxin's light chain has zinc-containing endopeptidase that blocks acetylcholine-containing vesicles from fusing with the terminal membrane of the motor neuron, resulting in flaccid muscle paralysis. Botulism can be classified into a number of groups; depending on the route the toxin enters the body like food borne botulism, infant botulism, wound botulism, hidden botulism, inadvertent botulism, and inhalational botulism. There were 197 outbreaks reported between 1920 and 2014., ${ }^{5,6}$ The goldstandard method has been proposed for BoNT/A detection in the mouse bioassay for studying its lethality test and it performed by injecting potential toxin-containing sample into live mice followed by observation for signs of botulinic paralysis or death. ${ }^{7-9}$ Other reported methods were also well known for detecting BoNTs have been reported, such as ELISA, surface plasma resonance (SPR) immunoassay, and electrochemical luminescence immunoassay. ${ }^{8-12}$ Among them, immunoassays method is more reliable for its lowest detection limit but require complicated operations and cumbersome instrumentation for achieving signal readouts. ${ }^{13-18}$ In recent years, electrochemical detection methods and Enzyme based biosensor based methods have proposed as most predominant analytical techniques for the easy quantification and simple instrumentation which designed or evolved for achieving most effective BoNTs detection in biological samples. ${ }^{19-23}$

\section{Most acclaimed methods used for BoNT detection}

Previously, most advanced biosensor has been proposed by using antibodies which is specific for Clostridium botulinum which was covalently attached to the surface of the tapered fiber using rhodamine-labeled polyclonal anti-toxin A immunoglobin G (IgG) antibodies for generation of the specific fluorescent signal. Affinity- 
purified polyclonal horse anti-toxin A antibodies performed better than the $\mathrm{IgG}$ fraction from the same horse serum or than the monoclonal anti-toxin A antibody BA11-3 nd BoNTs could be detected within a minute with lowest detection limit. ${ }^{14}$ Previously, an aptamer probe was used to develop electrochemical method to detect BoNT/A in which a substrate treptavidin-dendrimer-interface-polypyrrole used in conjugation with horseradish peroxidase (HRP) and an antifluorescein antibody. ${ }^{15}$ Electrochemical biosensor based methods are considered the most specific method for determination of BoNT which based on the principle of signal transduction. In these methods, BoNT-neurotoxin type A (BoNT/A) was fabricated onto glassy carbon electrode modified with Aunanoparticles/graphene-chitosan for signal amplification and improved specificity of assay. ${ }^{24}$

In other method, gold nanodendrites and chitosan nanoparticles(AuNDs/CSNPs) were fabricated onto carbon electrode to develop an impedimetric immunosensor to detect botulinum neurotoxin A (BoNT/A). In previous methods, BoNT/E and anexopeptidase, L-leucine-aminopeptidase (LAP) were used to develop electrochemical biosensor based on amplified signal through a redox cycling by involving a reducing agent and $\mathrm{Ru}(\mathrm{NH} 3) 6^{3+}$ as fluorogenic reporters. ${ }^{25}$

A robust fluorescence biosensor for the ultra-sensitive detection of Clostridium botulinum Neurotoxin Type A (BoNT/A) has been developed to achieve highly specific detection of neurotoxins by using photostable dye-doped nanoparticle (DOSNP) tags and high surface area nanoporous organosilicate (NPO) thin films conjugated with dye-labeled antibodies whose detection limit was compared with ELISA. And, it was observed a novel method for rapid, ultra-low level detection of not only BoNT/A as well as other biological and chemical analytes. ${ }^{26}$

A novel assay was reported earlier and found to be more specific when compared to other conventional methods of detection of Botulinum neurotoxins (BoNTs) in biological samples. It is utilized the endopeptidase activity of the toxin to detect BoNTs by using SNAP-25 whose cleaving is monitored via UV-Visible spectroscopy with a limit of detection of $373 \mathrm{fg} / \mathrm{mL}$ and has been further used to develop into a high throughput method using a microplate reader detecting down to $600 \mathrm{fg} / \mathrm{mL}$ of active toxin. This observation is found to be more fugitive to know the precise differences between the toxin product and the placebo. ${ }^{28}$

\section{Conclusion}

This short review is based on information of most rapid and reliable detection methods used for BoNTs which are necessary to support clinical diagnosis and surveillance to decrease the chances of botulism outbreaks in susceptible population. This fugitive and recent information would be helpful to develop more advanced biosensors to detect botulinum neurotoxins with enhanced sensitivity, specificity, reusable and lower detection limit. As well as, it can be further employed to carry out rapid screening of neurotoxins in various food samples with improved longevity to prevent havoc of its outbreak and effective clinical management.

\section{Acknowledgments}

None.

\section{Conflicts of interest}

The author declares that there is no conflicts of interest.

\section{References}

1. F Anniballi, A Fiore, C Löfström, et al. Woudstra et al. Management of animal botulism outbreaks: from clinical suspicion to practical countermeasures to prevent or minimize outbreaks. Biosecur Bioterror. 2013;11:191-199.

2. O Rossetto. Botulinum Toxins: Molecular Structures and Synaptic Physiology. Botulinum Toxin Treatment in Clinical Medicine. 2018:1-12.

3. FJ Erbguth. Historical notes on botulism, Clostridium botulinum, botulinum toxin, and the idea of the therapeutic use of the toxin. Mov Disord. 2004;19:S2-S6.

4. C Lamanna, The most poisonous poison. Science. 1959;130:763-772.

5. SS Arnon, R Schechter, TV Inglesby, et al. Botulinum toxin as a biological weapon- medical and public health management. J Am Med Assoc. 2001;285(8):1059-1070.

6. SK Sharma, BS Eblen, RL Bull, et al. Evaluation oflateral- flow Clostridium botulinum neurotoxin detection kits for food analysis, Appl. Environ. Microb. 2005;71:3935-3941.

7. MA Poli, VR Rivera, D Neal. Development of sensitive colorimetric captureELISAs for Clostridium botulinum neurotoxin serotypes $\mathrm{E}$ and F. Toxicon. 2002;40(6):797-802.

8. J Ladd, AD Taylor, J Homola, et al. Detection of botulinum neurotoxins in buffer and honey using a surface plasmon resonance (SPR) sensor. Sens Actuators B Chem. 2008;130:129-134.

9. VR Rivera, FJ Gamez, WK Keener, et al. Rapid detection of Clostridium botulinum toxins A, B, E, and F in clinical samples, selected food matrices, and buffer using paramagnetic bead- based electrochemiluminescence detection. Anal Biochem. 2006;353(2):248-256.

10. Grate JW, Ozanich RM, Warner MG, et al. Advances in assays and analytical approaches for botulinum- toxin detection. TrAC Trends in Analytical Chemistry. 2010;29(10):1137-1156.

11. G Ferracci, S Marconi, C Mazuet, et al. A label- free biosensor assay for botulinum neurotoxin B in food and human serum. Anal Biochem. 2011;410(2):281-288.

12. ML Frisk, G Lin, EA Johnson, et al. Synaptotagmin II peptide- bead conjugate fo rbotulinum toxin enrichment and detection in microchannels, Biosensors and Bioelectronics. 2011;26:1929-1935.

13. P Pashazadeh, A Mokhtarzadeh, M Hasanzadeh, et al. Nano- materials for use in sensing of salmonella infections: Recent advances. Biosens Bioelectron. 2017;87:1050- 1064.

14. RA Ogert, JE Brown, BR Singh, et al. Detection of Clostridium botulinum toxin A using a fiber optic- based biosensor. Analytical Biochemtry. 1992;205(2):306-312.

15. F Wei, CM Ho. Aptamer- based electrochemical biosensor for Botulinum neurotoxin. Anal Bioanal Chem. 2009;393(8):1943-1948.

16. MS Wilson. Electrochemical immunosensors for the simultaneous detection of two tumor markers. Anal Chem. 2005;77(5):1496-1502.

17. R Sorouri, H Bagheri, A Afkhami, et al. Fabrication of a Novel Highly Sensitive and Selective Immunosensor for Botulinum Neurotoxin Serotype A Based on an Effective Platform of Electrosynthesized Gold Nanodendrites/Chitosan Nanoparticles. Sensors. 2017;17(5):1074.

18. J Narayanan, MK Sharma, S Ponmariappan, et al. Electrochemicalimmunosensor for botulinum neurotoxin type- E using covalently ordered graphenenanosheetsmodified electrodes and gold nanoparticles- enzyme conjugate. Biosens Bioelectron. 2015;69:249256.

19. G Liu, Y Zhang, W Guo. Covalent functionalization of gold nanoparticles 
as electronic bridgesand signal amplifiers towards an electrochemical immunosensor for botulinum neurotoxin type A. Biosens Bioelectron. 2014;61:547-553.

20. Boyer AE, Moura H, Woolfitt AR, et al. From the mouse to the mass spectrometer: Detection and differentiation of the endoproteinase activities of botulinum neurotoxins A-G by mass spectrometry. Anal Chem. 2005;77:3916-3924.

21. Kalb SR, Moura H, Boyer AE, et al. The use of Endopep- MS for the detection of botulinum toxins A, B, E, and F in serum and stool samples. Anal Biochem. 2006;351(1):84-92.

22. Sharma SK, Ferreira JL, Eblen BS, et al. Detection of type A, B, E, and $\mathrm{F}$ Clostridium botulinum neurotoxins in foods by using an amplified enzyme- linked immunosorbent assay with digoxigenin- labeled antibodies. Appl Environ Microbiol. 2006;72(2):1231-1238.

23. Zhang Y, Lou J, Jenko KL, et al. Simultaneous and sensitive detection of six serotypes of botulinum neurotoxin using enzyme- linked immunosorbent assay- based protein antibody microarrays. Anal Biochem. 2012;430(2):185-192.
24. Lindström M, Keto R, Markkula A, et al. Multiplex PCR assay for detection and identification of Clostridium botulinum types A, B, E, and F in food and fecal material. Appl Environ Microbiol. 2001;67(12):5694 5699.

25. Ruge DR, Dunning FM, Piazza TM, et al. Detection of six serotypes of botulinum neurotoxin using fluorogenic reporters. Anal Biochem. 2011;411(2):200-209.

26. Bok S, Korampally V, Darr CM, et al. Femtogram- level detection of Clostridium botulinum neurotoxin type $A$ by sandwich immunoassay using nanoporous substrate and ultra- bright fluorescent suprananoparticles. Biosensors and Bioelectronics. 2013;41:409-416.

27. Halliwell J, Savage AC, Buckley N, et al. Electrochemical impedance spectroscopy biosensor for detection of active botulinum neurotoxin. Sensing and Bio-Sensing Research. 2014;2:12-15.

28. Halliwell J, Gwenin C. A label free colorimetric assay for the detection of active botulinum neurotoxin type A by SNAP- 25 conjugated colloidal gold. Toxins. 2013;5:1381-1391. 